\title{
Gradient RSSI Filter and Predictor for Wireless Networks Algorithms and Protocols
}

\author{
Alvaro Suárez, Kholoud Atalah Elbatsh and Elsa Macías \\ Concurrency and Architecture Group (GAC) \\ Department of Telematic Engineering \\ University of Las Palmas de Gran Canaria \\ Spain
}

asuarez@dit.ulpgc.es, kholoud.elbatsh101@doctorandos.ulpgc.es, emacias@dit.ulpgc.es

Received: June 15, 2010 Accepted: July 14, 2010 DOI: 10.5296/npa.v2i2.414

\begin{abstract}
Wireless networks, and in particular Wireless Fidelity (WiFi) networks, present important problems of connectivity among wireless terminals. These problems lead to inefficiencies in the Application level. For localization algorithms in wireless networks, efficient handover protocols and multimedia delivery information, these problems must be solved. A figure used to control the connectivity of terminals to other terminals or to an Access Point (AP) is the well known Received Signal Strength Indicator (RSSI). Managing this figure it is possible to control the availability of a service in the wireless terminal. But the problem with RSSI is the fact that it exhibits high variability in space and time. Thus it is important to filter those values in order to efficiently manage the availability of the service in the mobile terminal. For that reason in this paper we present the experimental validation of a filter and a predictor of availability of a service in the wireless terminal based on the RSSI. We test the performance of the filter and the predictor that improves significantly other filters that are also presented in the paper. The significance of our work is that our filter allows simplifying a lot of network algorithms and protocols. As an example we present a simple protocol of handover in presence of multimedia information using our filter and predictor based on RSSI.
\end{abstract}

Keywords: Handover prediction, RSSI gradient, RSSI filters, Kalman filter, Grey model. 


\section{Introduction}

It is well known that the behavior of the physical wireless channels experiments high variations in time and space. This is true for mobile terminals (Mobile Client $(M C)$ from now on), or a Static Client (it moves but relatively low or indeed it does not move). For high frequencies $(10 \mathrm{GHz})$ weather conditions could influence the variation of the wireless channel conditions (wind, temperature ...). For low frequencies, parameters like fading, interferences, and the electronic of MC's Wireless Network Card Interface (WNIC) ... could influence those variations.

The principal consequence of the above variations is that the MC experiments disconnections to the AP or to another MC. As a result data packets will be lost. A very important associated problem is the MC cannot differentiate loss of packets from data packets errors. This produces a high loss of effective bandwidth in the channel that greatly influences the performance of the channel.

A parameter used to test the level of connectivity of the MC to the AP is the RSSI. RSSI increases if the MC will be in a good connection state. A low level parameter normally used to evaluate the connectivity is the Signal Noise Ratio (SNR). With this and other low level parameters it is possible to exactly define the state of the MC and its variations. But with RSSI that accuracy cannot be assured. Moreover, different WNIC's drivers defined the RSSI in different scales. That is, the driver also influences the measurement of the RSSI. For this reason it is normal to use the RSSI\% that is given in percentage values. RSSI\% can be calculated by dividing the real measured RSSI over the maximum value of RSSI assigned to WNIC by its manufactured company.

Some works have been done to control these variations or to mitigate the effects of these variations. The technique consists of the design of a filter for the RSSI values to smooth its variation. A lot of filters have been designed. Grey Model (GM), Kalman filter, Fourier transform, particle [1] and Bayesian filter [2] are normally used by many researches. Among them, the GM and the Kalman filter are two important ones because they allow predicting values of RSSI inferring its value taking into account some past values of RSSI in a very simple way. To predict the future values of RSSI is very important because it allow Application services to adapt its behavior to wireless channel variations.

These filters and predictors can be used in a variety of different applications (we present them in section 4). For example, they translate the values of RSSI in geographical positions [3], estimate the location and velocity of a tracked MC [4], or apply results to handover process [5]. All the above works suppose that it is possible to define different homogeneous (all their internal points have the same level of RSSI) regions in the coverage area taking into account the RSSI values for the different positions. Recently in [6] were defined these areas and linear path are simulated to adapt the video rate from a video server to a streaming client. But in practice, the homogeneity does not exist. Inside areas there are holes (zones of the coverage area in which suddenly the coverage change enormously to fall down to no coverage state of connectivity). We think to take into account a hole is very important due to it causes suddenly the degradation of the bandwidth causing problems to the wireless service. 
In this paper we present the validation o our previously designed RSSI prediction technique [7] that is inspired on the Gradient mathematical figure. We consider the MC is moving with constant speed inside the coverage area defining different kinds of movements (not only linear ones). We have supposed a set of paradigmatic kind of movements and an uniform distributed signal strength inside the coverage area but with holes. Theoretically, our new predictor is developed to support shortly disconnections when the MC fall in one of these holes. The objective is to improve the behavior of GM and Kalman filters for short term disconnections. Results from synthetic values of RSSI\% show that our filter and predictor behaves better than the GM and Kalman filters in presence of holes. For that reason we only validate our filter experimentally.

The rest of the paper is organized as follows: in Section 2, Grey Model, Kalman filter, and our developed Gradient RSSI predictor and filter are presented from the theoretical point of view. Section 3 presents an experimental evaluation of our Gradient RSSI filter and predictor. In Section 4 we present the applications of our filter and predictor in three areas. Finally, we summarize our conclusions and present directions for further research.

\section{Performance of Grey model, Kalman filter and Gradient filter}

In this section we briefly present some characteristics of the GM and Kalman filters. Then we present our Gradient RSSI filter and predictor. Finally a theoretical comparison among them is presented.

\subsection{Grey Model}

In the System Theory, a White System is defined as that for which all information about it is known. On the contrary, a Black System (also known as Black Box model) is defined as that for which no information about it is known [8] [5]. In the middle of those kinds of systems is the Grey System (also named Grey model). A Grey model is defined as that, for which only partial information about it is known [5] [9]. The Grey System mainly works on a system analysis which has produced poor, incomplete or uncertain messages. One advantage of a GM is that it allows using a few data to estimate an unknown system. Another advantage is its mathematical modeling simplicity. The unknown system's behavior can be modeled by a first-order differential equation [8]. The most commonly used Grey Model is the GM $(1,1)$, that is a single variable first-order Grey Model.

The idea is we know partial information about the RSSI\% of the MC in a coverage zone. We are interested in to predict the value of RSSI\% of the MC when it will move inside the coverage area. Then we can use the GM to predict those values. The basic idea is to consider a set of values of RSSI\% are the input of the Grey System, we know a law of behavior of the Grey system and then we will compute an output that are the predicted values of RSSI\%.

The modeling procedure is as follows: 
1. Given the original data set: $\operatorname{RSSI}^{0}{ }^{0}=\left[\operatorname{RSSI} \%^{0}(1), \operatorname{RSSI} \%^{0}(2), \ldots ., \operatorname{RSSI} \%^{0}(n)\right]$, where $R S S I \%^{0}(i)$ corresponds to the system input at time $i$.

2. A new sequence $\quad R S S I \%^{1}=\left[R S S I \%^{1}(1), R S S I \%^{1}(2), \ldots ., R S S I \%^{1}(n)\right]$ is generated, where $\operatorname{RSSI} \%^{1}(k)=\sum_{m=1}^{k} R S S I \%{ }^{0}(m)$

3. From $R S S I \%^{1}$ we can form the first-order differential equation $\frac{d R S S I \%^{1}(k)}{d k}+a R S S I \%^{1}(k)=u$

4. From which it is possible to obtain $\boldsymbol{a}$ and $\boldsymbol{u}$ with $\left[\begin{array}{l}a \\ u\end{array}\right]=\left(B^{T} B\right)^{-1} B^{T} R S S I \%^{\prime}{ }_{n}$ Where:

$$
B=\left[\begin{array}{l}
-1 / 2\left(R S S I \%{ }^{1}(1)+R S S I \%{ }^{1}(2)\right) \\
-1 / 2\left(R S S I \%{ }^{1}(2)+R S S I \%{ }^{1}(3)\right) \\
\cdots . . \\
-1 / 2\left(R S S I \%{ }^{1}(n-1)+R S S I \%{ }^{1}(n)\right)
\end{array}\right]
$$

And

$$
\operatorname{RSSI} \%_{n}^{\prime}=\left[\operatorname{RSSI} \%^{0}(2), \operatorname{RSSI}^{0}{ }^{0}(3), \ldots ., \operatorname{RSSI} \%^{0}(n)\right]^{T}
$$

5. The predictive function is $\hat{R S S I} \%^{1}(k)=\left(R S S I \%^{1}(1)-u / a\right) e^{-a k}+u / a$ and the predicted value at time $k+1$ is $\hat{R S S I} \%^{0}(k+1)=\hat{R} S S I \%{ }^{1}(k+1)-\hat{R} S S I \%{ }^{1}(k)$ which can be written as:

$$
\hat{\operatorname{RSSI}} \%^{0}(k+1)=\left(\operatorname{RSSI} \%^{0}(1)-u / a\right)\left(e^{-a}-1\right) e^{-a k}
$$

Which corresponds to the predicted value of RSSI\% considering the inputs values of step one. 
In this simple way and dimensioning appropriately the values of the parameters $a$ and $u$ we can predict this value of RSSI\%.

\subsection{Kalman Model}

We understand for Kalman model the formulation of a Kalman Filter for modeling a system in which we know a set of values of RSSI\% and want to predict the values for a future value of time knowing some information of the system.

The Kalman filter is a set of mathematical equations that provides an efficient computation (recursive) mechanism to estimate the state of a process, in a way that minimizes the mean of the squared error. The filter is very powerful in several aspects: it supports estimations of past, present, and even future states, and it can do so even when the precise nature of the modeled system is unknown [10].

We apply the Discrete Kalman filter trying to estimate RSSI\% values by representing the RSSI\% time evolution as a combination of signal noise (measurement noise) and maximum signal evolving (process noise) like in [1]. To formulate our Kalman filter we will name the variable RSSI\% using another notation ( $x$ in general) in order to simplify the understanding if the formulation.

The filter is used to estimate the state $x \in R^{n}$ of a discrete time controlled process, $\hat{x}_{k}^{-} \in R^{n}$ is a Priori state estimate at step $k$, given knowledge of the process prior to step $k$, and $\hat{x}_{k} \in R^{n}$ is a Posteriori state estimate at step $k$ given measurement $z_{k}$.

The Discrete Kalman filter algorithm consists of two phases:

1. Time update (Predictor): Projecting forward the current state and error covariance estimations to obtain a priori estimation for the next time step.
a. To project the state ahead
$\hat{x}_{k}^{-}=A \hat{x}_{k-1}+B u_{k-1}$
b. Project the error covariance ahead
$P_{k}^{-}=A P_{k-1} A^{T}+Q$

2. Incorporating a new measurement into a priori estimation to obtain an improved a posteriori estimation.
a. Compute the Kalman gain:
$K_{k}=P_{k}^{-} H^{T}\left(H P_{k}^{-} H^{T}+R\right)^{-1}$
b. Update estimation with measurement
$z_{k}, \quad \hat{x}_{k}=\hat{x}_{k}^{-}+K_{k}\left(z_{k}-H \hat{x}_{k}^{-}\right)$ 
c. Update the error covariance: $\quad P_{k}=\left(1-K_{k} H\right) P_{k}^{-}$

As initial step for the first state, initial estimations for $\hat{x}_{k-1}$ and $P_{k-1}$ used as inputs:

i. Let $A(n \times n)$ be a matrix that relates the step of $k-1$ to current state $k$

ii. Let $B(n \times 1)$ be a matrix that relates $u$ to the state $x$

iii. Let $u$ the optional control input

iv. Let $P_{k}^{-}$: be a priori estimation error covariance

v. Let $Q$ be a process noise covariance

vi. With a real measurement $z \in R^{m}$ that is: $z_{k}=H x_{k}+v_{k}$

vii. Let $v_{k}$ be a random variable represents a measurement noise that has normal probability distribution $p(v) \approx N(0, R)$

viii. Let $R$ be the measurement noise covariance. Notice that While $Q$ and $R$ constant $\rightarrow P_{k}$ and $K_{k}$ are constant.

ix. Let $H(m \times n)$ be a matrix that relates the state $x$ to the measurement $z_{k}$.

We, like [1], consider the following assumptions:

i. Let be $A=I$ if the state will not change from step to step, where $I$ is the identity matrix.

ii. Let be $u=0$ (there is not control input).

iii. Let be $Q=0$ (assumed to be very small).

iv. Let be $R=0.01$ (in [11] it is used $Q / R$ as standard deviation, and $Q$ is the initial state of $P_{k}$ ). 
v. Let be $H=I$ is a noisy measurement of the state directly. Then:

$$
\begin{aligned}
& x_{k}=x_{k-1}+w_{k-1} \\
& z_{k}=x_{k}+v_{k}
\end{aligned}
$$

With all previous assumptions we obtain the following equations:

1. Time update:

$$
\begin{aligned}
& \hat{X}_{k}^{-}=\hat{X}_{k-1} \\
& P_{k}^{-}=P_{k-1}+Q
\end{aligned}
$$

2. Measurement update:

$$
\begin{aligned}
& K_{k}=P_{k}^{-}\left(P_{k}^{-}+R\right)^{-1} \\
& \hat{x}_{k}=\hat{x}_{k}^{-}+K_{k}\left(z_{k}-\hat{x}_{k}^{-}\right) \\
& P_{k}=\left(1-K_{k}\right) P_{k}^{-}
\end{aligned}
$$

Solving the filter equations we obtain the following two equations to be used in the RSSI filtering:

$$
\begin{gathered}
P_{k}=\frac{R\left(P_{k-1}+Q\right)}{P_{k-1}+Q+R} \\
\hat{x}_{k}=\hat{x}_{k-1}+\frac{\left(P_{k-1}+Q\right)\left(z_{k}-\hat{X}_{k-1}\right)}{P_{k-1}+Q+R}
\end{gathered}
$$

This corresponds to the predicted value of RSSI\% at time $\mathrm{k}$ considering the values of step k-1. In this simple way and dimensioning appropriately the values of the parameters of the filter (as indicated) we can predict this value of RSSI\%. 


\subsection{Gradient filter modeling}

In [7] we presented the concept and some graphics explaining our Gradient filter. Next we present the basic idea and the modeling of our Gradient filter.

The gradient of the RSSI\% is defined over a coverage area scalar field that is a two dimensional space with a real RSSI value associated to each point inside the field. Associated to this scalar field is a vector field defined by the gradient of two different values of RSSI in the scalar field. The gradient of RSSI vary over time and space, that is, if the MC change its position in the scalar field the gradient will vary, and if the MC is static but the RSSI change over time the gradient will also change.

We consider an initial value of RSSI\% for the MC as an entry to our filter. After a prediction process the filter will output the prediction of the next RSSI\% value of the MC after a certain amount of time. Next, we present the prediction process and then we will justify the Gradient filter in order to adjust certain kinds of movements of the MC.

\subsubsection{The prediction process}

To simplify the analysis let us suppose that the MC is moving. The nature of the movement is not important for our analysis. But let us suppose the speed of the MC is constant. Let be the vector $x=\left[x_{0}, x_{1}, \ldots \ldots ., x_{n}\right]$, a set of $n$ values of RSSI\% measured in the interval $t$ of time.

Where $x_{0}$ is measured at time $t_{0}$, and $x_{n}$ is measured at time $t$.

Let us now define The Instant Gradient of RSSI\% at any time as:

$$
\nabla x_{k}=x_{k}-x_{k-1}
$$

With this figure, applying this formula to all pairs of values of RSSI\%, we obtain the following vector:

$$
\nabla x=\left[\nabla x_{1}, \nabla x_{2}, \ldots \ldots ., \nabla x_{n}\right]
$$

Let us now define The Instant Gradient of RSSI\% at instant of time $t$ as:

$$
\nabla\left(x_{k}\right)_{t}=\frac{\nabla x_{k}}{\Delta t_{k}}
$$

Where: $\Delta t_{k}=t_{k}-t_{k-1}$.

The average gradient with respect to time after interval of time equal $t$ is: 


$$
\nabla\left(x_{n}\right)_{t}=\frac{\nabla x_{n}}{\Delta t}
$$

Where: $\nabla x_{n}=x_{n}-x_{0}$ and $\Delta t=t-t_{0}$.

Then, if $x_{n}$ was unknown, it can be calculated from the following equation if all the other variables are known:

$$
x_{n}=\nabla\left(x_{n}\right)_{t} \times \Delta t+x_{0}
$$

To calculate $x$ at any time $t_{k}$, the same equation can be used changing the interval of time and we will call this value of $x$ the predicted value:

$$
\bar{x}_{k}^{-}=\left(\nabla\left(x_{n}\right)_{t} \times \Delta t\right)+x_{0}
$$

Where: $\Delta t=t_{k}-t_{0}$.

Taking into account that an average gradient equals the average of the summation of instants gradient in the same interval of time:

$$
\nabla\left(x_{n}\right)_{t}=\frac{1}{n} \sum_{k=1}^{n} \nabla\left(x_{k}\right)_{t}
$$

Then the predicted value of $x$ will be:

$$
\begin{gathered}
\hat{X}_{k}^{-}=\left(\nabla\left(x_{n}\right)_{t} \times \Delta t\right)+x_{0} \\
\hat{x}_{k}^{-}=x_{0}+\frac{\Delta t}{n} \sum_{k=1}^{n} \nabla\left(x_{k}\right)_{t} \\
\hat{x}_{k}^{-}=x_{0}+\frac{\left(t_{k}-t_{0}\right)}{n} \sum_{k=1}^{n}\left(\frac{x_{k}-x_{k-1}}{t_{k}-t_{k-1}}\right)
\end{gathered}
$$

Any RSSI value can be estimated using this formula, exactly at the time we want to know it.

\subsubsection{The filtering process}




\section{IIMacrothink

In some cases, when the RSSI gradient is increasing and decreasing sequentially, the formula of Gradient predictor is no longer giving correct estimation for all kinds of movements. For this reason, it is important to take in mind the error of estimation between the current predicted value and the previous measured value. This error is calculated using the standard deviation as:

$$
\sigma\left(x_{k-1}, \hat{x}_{k}^{-}\right)=\sqrt{\frac{1}{2}\left(x_{k-1}-\hat{x}_{k}^{-}\right)^{2}}
$$

By adding or subtracting this standard deviation value to the predicted value, the estimated values of RSSI will be:

$$
\bar{X}_{k}=\widehat{X}_{k}^{-} \pm \sigma\left(x_{k-1}, \widehat{X}_{k}^{-}\right)
$$

For each case of $x$ adding or subtracting the value of the standard deviation must be decided. This can be solved by multiplying the standard deviation by the SIGN function (SIGN of the difference between the two inputs of the standard deviation), because the SIGN function gives 1 if the input will be positive and gives -1 if it will be negative. Then the formula of the estimated value will be:

$$
\hat{x}_{k}=\hat{x}_{k}^{-}+\operatorname{SIGN}\left(x_{k-1}-\hat{x}_{k}^{-}\right) \cdot \sigma\left(x_{k-1}, \hat{x}_{k}^{-}\right)
$$

Applying this formula to RSSI\% values which include zero values for holes, the formula does not estimate correct value.

One solution to consider the holes is to reformulate the formula in two steps:

i. To find a condition that detects the zero values. This can be solved by multiplying the previous measured value by the current value; if the result is zero, one of them will be zero.

ii. Removing the effect of the error part from the formula if the first condition will be achieved without changing the original value.

To insert these conditions in the model, to multiply by zero is required if the first condition will be achieved or to multiply by 1 if it will not achieve. One function that solves this is the SIGN function:

$$
\operatorname{SIGN}\left(x_{k-1} \cdot x_{k}\right)^{2}=\left\{\begin{array}{l}
0 \\
1
\end{array}\right.
$$


The final formula of the filtered predicted (estimated) value:

$$
\hat{X}_{k}=\hat{X}_{k}^{-}+\operatorname{SIGN}\left(x_{k-1}-\hat{X}_{k}^{-}\right) \cdot \sigma\left(x_{k-1}, \hat{X}_{k}^{-}\right) \cdot \operatorname{SIGN}\left(x_{k-1} \cdot x_{k}\right)^{2}
$$

This corresponds to the predicted value of RSSI\% at time $\mathrm{k}$ considering the values of step $k-1$ and the presence of holes. In this very simple way the value of RSSI\% is predicted and filtered in order to consider holes inside the coverage area.

\subsection{Comparison of the Theoretical Performance}

We did a lot of probes for the different filters introducing in a simple simulator (basically implementing the different formulas of the filters) a lot of synthetic values of RSSI\%. We consider a lot of kinds of movements and holes inside the coverage area and reflect them into the corresponding values of synthetic values of RSSI\%.

In table 1, a qualitative evaluation for the three filters performance is shown. We use different numbers to indicate the comparison. Numbers 1, 2, 3, and 4 are used where 1 means the best filter, 2 means good, 3 means the worst filter, and 4 means the filter does not work. For prediction accuracy we understand how close is the filter output to the synthetic value of RSSI\%. In the row named kind of movement is presented the path that is represented by synthetic values of RSSI\%. For example, let us suppose the movement named forward to the AP. This movement is the one in which we suppose the MC is moving from the geographical point of the AP to the far geographical point (limit of the coverage area). To do that, we choose a set of values of RSSI\% from the theoretical behavior of RSSI in the coverage area. Then we introduced then into a spreadsheet and finally we select a formula that implements the computation of the different filters. In the same spreadsheet we compared the theoretic performance of the filters obtaining the comparison data of table 1.

In general, the Gradient RSSI filter exhibits the best behavior for holes and for certain kind of movements it is the better in prediction accuracy. For same particular kinds of movements the Kalman filter exhibit better accuracy, when there is no holes along the path of the MC. Also the GM has particular better performance when there are no holes along the path of the MC. 
Table 1: Filters Qualitative Comparison.

\begin{tabular}{|c|c|c|c|}
\hline Kind of movement & Filter & $\begin{array}{c}\text { Holes detection } \\
\text { and support }\end{array}$ & $\begin{array}{r}\text { Prediction } \\
\text { accuracy }\end{array}$ \\
\hline \multirow{3}{*}{ Backward to the AP } & Kalman & 3 & 2 \\
\hline & $G M$ & 4 & 3 \\
\hline & Gradient & 1 & 1 \\
\hline \multirow{3}{*}{ Forward to the AP } & Kalman & 3 & 2 \\
\hline & $G M$ & 4 & 3 \\
\hline & Gradient & 1 & 1 \\
\hline \multirow{3}{*}{ Along a circumference } & Kalman & 3 & 1 \\
\hline & $G M$ & - & - \\
\hline & Gradient & 1 & 1 \\
\hline \multirow{3}{*}{ Sine along a circumference } & Kalman & 2 & 2 \\
\hline & $G M$ & 4 & 3 \\
\hline & Gradient & 1 & 1 \\
\hline \multirow{3}{*}{ Zigzag along a circumference } & Kalman & 3 & 2 \\
\hline & $G M$ & 4 & 3 \\
\hline & Gradient & 1 & 1 \\
\hline \multirow{3}{*}{$\begin{array}{l}\text { Spiral at a distance from the } \\
\qquad A P\end{array}$} & Kalman & 3 & 2 \\
\hline & $G M$ & 4 & 3 \\
\hline & Gradient & 1 & 1 \\
\hline \multirow{3}{*}{$\begin{array}{l}\text { Irregular movement crossing } \\
\text { different levels of RSSI }\end{array}$} & Kalman & 3 & 1 \\
\hline & $G M$ & 4 & 3 \\
\hline & Gradient & 1 & 2 \\
\hline
\end{tabular}

\section{Experimental Validation of RSSI Gradient}

In section 2 we presented the comparison of the three filters: GM, Kalman and Gradient RSSI filter and predictor. In this section we will present some experimental measures we did outdoors in order to verify that in some cases the holes inside the coverage area exist and impose several problems of performance to the filters and predictors. We only test the Gradient RSSI filter and predictor because it is the better in the above comparison.

\subsection{The coverage area properties and physical environment}

The signal scanning experiment was done in an open area with no obstacles or buildings to avoid their effects. The coverage area was studied in the football camp of the University of Las Palmas de Gran Canaria (ULPGC). The straight line was the shape of movement, because MC is moving with constant speed $1 \mathrm{~m} / \mathrm{s}$ and it was the easiest shape for human walking. Eight directions are considered on the ground of the camp (Figure 5.1), in each direction MC scanned the signal 4 times backward (far from) the AP and 4 times forward to 
the AP. In total, 192 groups of values were measured in 3 days. Directions that are shown in Figure 1 are as follows:
i. $\quad G S-A$ : Goal Soccer A.
ii. $\quad G S-B$ : Goal Soccer B.
iii. $\quad F$ : Front.
iv. $\quad S$ : Seats.
v. F-GS-A: Diagonal direction between the GS-A and F.
vi. F-GS-B: Diagonal direction between the GS-B and F.
vii. $\quad S-G S-A$ : Diagonal direction between the GS-A and S.
viii. $\quad S-G S-B$ : Diagonal direction between the GS-B and S.
ix. FOR: Forward the AP.
x. $\quad$ BAC: Backward (far from) the AP.

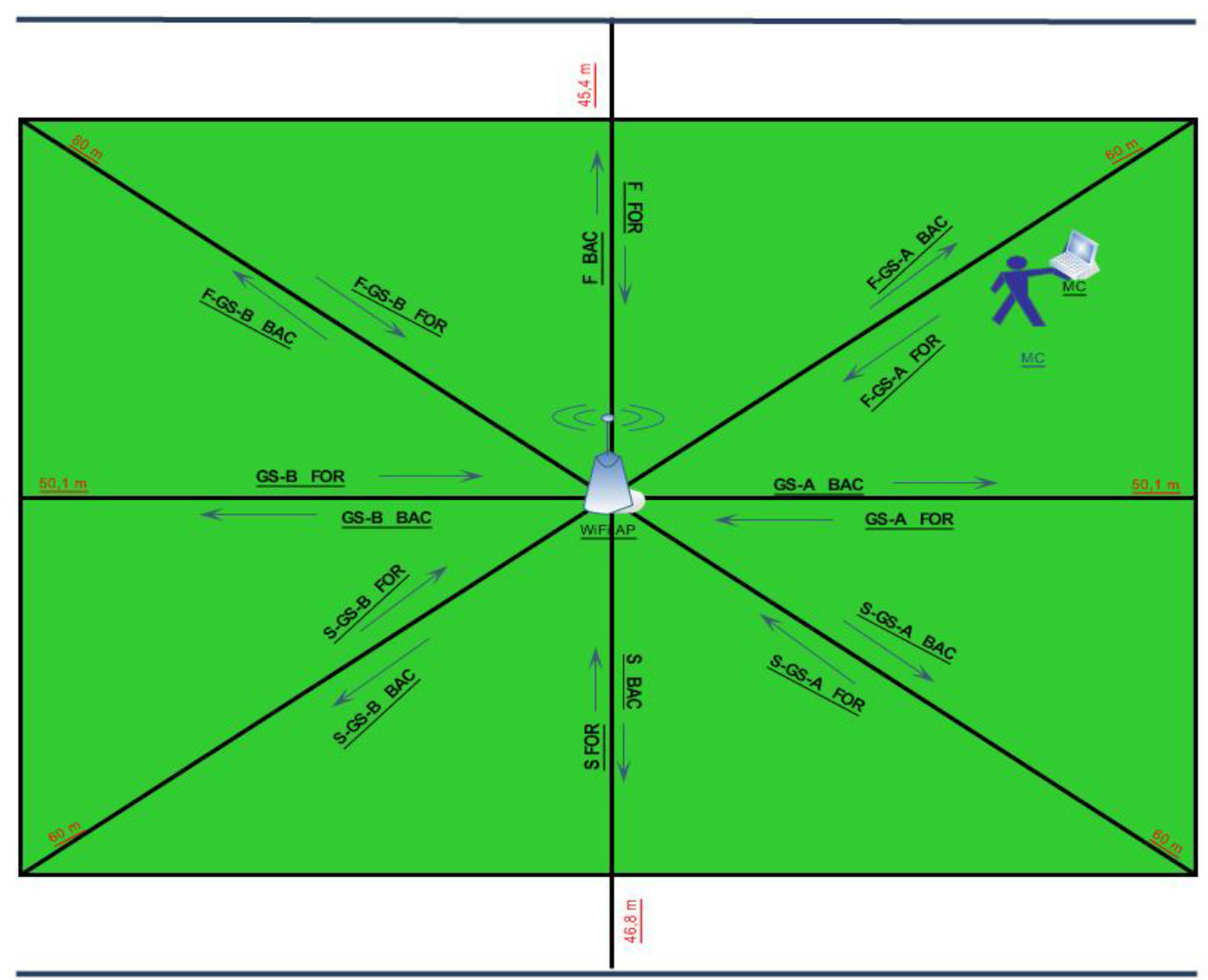

Figure 1: Football camp of ULPGC 
The measurements were done in different hours each day, the weather was sunny sometimes and cloudy in others days.

The equipments we used are the following: The AP used was ANSONIC USB wireless adapter (model number: AN-W541USB) which works as an AP or wireless adapter; it can be connected to any laptop in the center of the football camp. The MC was a laptop Sony VAIO PCG-TR5MP with WNIC Intel(R) PRO/Wireless 2200BG Network Connection (model number: WM3B2200BG). The Network Stumbler Version 0.4.0 software was installed on laptop to scan the signal on Windows XP operating system.

In Network Stumbler, the scanning frequency only is controlled by speed controller range between slow and fast, we could not determine exactly the speed of scanning. In the actual scanning results it gave 2 scanning each 1 second, and we was interested in scanning frequency 1scan/s so we calculated the medium of each two measurements.

The Network Stumbler program exported a data file that contains the signal values as RSSI (an integer and may be in the range 0-255). We used a mapping method to convert these integer values to RSSI\% values by taking maximum RSSI value $\left(R S S I \_M a x=122\right)$, and the minimum (RSSI_Min $=0$ ), which was obtained during all measurements, in order to calculate RSSI\% by the formula:

$$
R S S I \%=\frac{R S S I}{\left(R S S I_{-} M a x-R S S I_{\_} \text {Min }\right) / 100}
$$

Due to we had 192 groups of values, we preferred to calculate the medium of values in the repeated directions of each day, for example we had 4 times of scanning in the direction SG-A (FOR) in the first day, we calculated the medium of these four values, as a result 2 groups of values still in each direction for each day, last values in total are reduced to 48 group (3 days, 8 directions, 2 groups FOR and BAC).

\subsection{Some interesting experimental measurements}

In this section we present some interesting experimental measurements in order to validate the application of our Gradient RSSI filter and predictor to real values of RSSI\%.

It is important to establish the difficulties and problems found in the football camp:

i. The coverage area is not circular. It has the typical aspect of an omni-directional antenna radiation coverage area.

ii. The above produced that the signal drops to low values of RSSI\% in a lot of parts of the coverage areas. That is, a lot of holes were found in the coverage area. 


\section{Macrothink}

iii. In addition to the consideration ii, in practice a lot of APs were detected as active in the coverage area and all of them in the same channel. This produced a

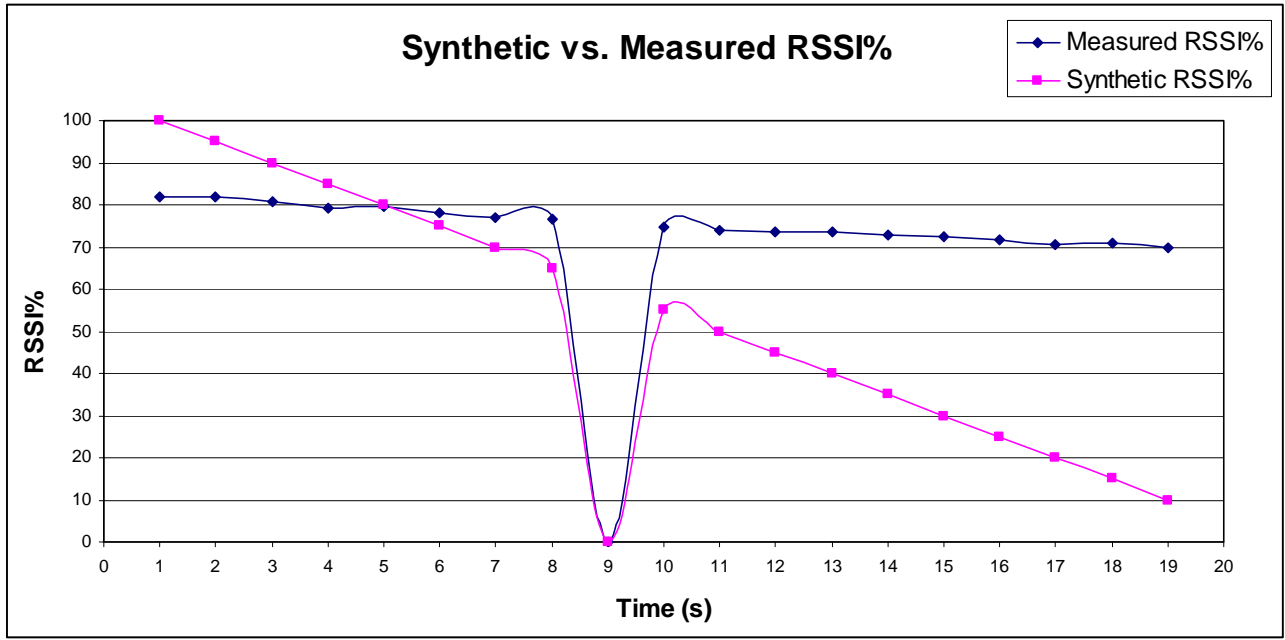

Figure 2: Comparison between synthetic \& measured RSSI\%

lot of interferences that also produce holes in the coverage area due to collocated radios.

iv. In the limit of the coverage area are collocated seats for people in one part, and an open space (aerial) in the opposite side due to there is a border of a river there. There is a lot of signal there that is lost due to there is no signal's reflections.

V.

We did the tests in three different days at sunset (18:00 hour). In the first two days the AP was set on $40 \mathrm{~cm}$ of height above the ground, on $40 \mathrm{~cm}$ of height above the ground, vertically oriented position to seats. In the third day it was set at $45 \mathrm{~cm}$ above the ground. In the first two days 16 APs were found in the third day 17 APs were found. The first day was clear (no wind). The second day was partially cloudy, North wind. The third day was partially cloudy and there was some winds low speed.

Taking into account the above consideration for actual experimentation, the first fact is that the experimentation is very hard due to the variability of measured signals that varied with the days and randomly due to the presence of other APs.

The first thing to show is the differences among the synthetic values and the actual values measured. In general the differences varied with the path of the MC. In Figure 2 we show the differences for the S-GS-A (BAC) movement. We highlight some interesting considerations:

i. In the border of the coverage area there was not possible to do a lot of measures. 


\section{$\triangle$ Macrothink}

ii. Closed to the AP the coverage is not $100 \%$ as we supposed for the synthetic values. The actual values are around $80 \%$.

iii. The general behaviors of the both signals are the same globally. The only that change is the slope of the straight lines.

iv. $\quad$ A hole is produce in the same place in both cases. It is important to note that in other paths the holes were not the same in both cases.

A very important problem is the variability of signal measured in different days (indeed in the same day) due to the consideration we exposed previously. We did a lot of measures but it is important to process them before to try to apply the test for the validation of the Gradient RSSI Gradient filter and predictor. First we must choose a representative set of values for the same experiment. Then it is necessary to process them in order to observe their global or shape variability.

For doing this we chose the graphic representation, used for the study of problems of prediction, also called regression analysis, of trends in the data series, such as a line sloping upward to represent values of RSSI\%. This trend must be referred to a parameter named coefficient of determination that in our case is a square value represented as $R^{2}$. This is a number in the interval $[0 . .1]$ that reveals how closely the estimated values for the trend correspond to the actual data. A trend is most reliable when its $R^{2}$ value is at or near 1 . We used two tests to discover the trend of the RSSI signal: linear (LIN) and exponential (EXP). It is important to discover this trend due to it influences the behavior of the Gradient RSSI filter and predictor.

As an example, in Figure 3 we show the trend of G-S-A (FOR) path for linear and exponential tests. Let us note that $m 1$ indicates the medium of measures of the first day, $m 2$ is the medium of second day and $m 3$ is the medium of the third day. The values of $R^{2}$ for the three days measures are also shown (from left to the right). Comparing the values of $R^{2}$ for both cases is simple to observe that the trend adapts well to the linear or exponential cases. We did the same for the S (BAC) path but it can be observed that in this case the linear test is the better.

The final step is to observe the behavior of the Gradient RSSI filter and predictor for these set of chosen values of RSSI putting special attention to holes. We first process then to obtain RSSI\% as indicated. In general all the paths that include the BAC direction (also all the paths that include FOR direction) exhibit in the final instants of time (initial for FOR direction) a hole. This hole (also if the number of holes is greater than one) and other sparse distributed along the path, with smooth variation of RSSI\%, are always detected by the Gradient RSSI filter and predictor as indicated in Figure 4.a (in actual experiments the predictor can anticipate the RSII\% till $45 \mathrm{~s}$ ). But there is still some sporadic cases in which the Gradient RSSI filter produce values not according with the actual values of RSSI\%. An example of this is shown in Figure 4.b. It is shown that in instant of time 78 there is a hole after a smooth variation of the RSSI\%. The filter produce an unexpected high value but the predictor produces a value that is according with the global slope of the RSSI\% curve. 
(a)
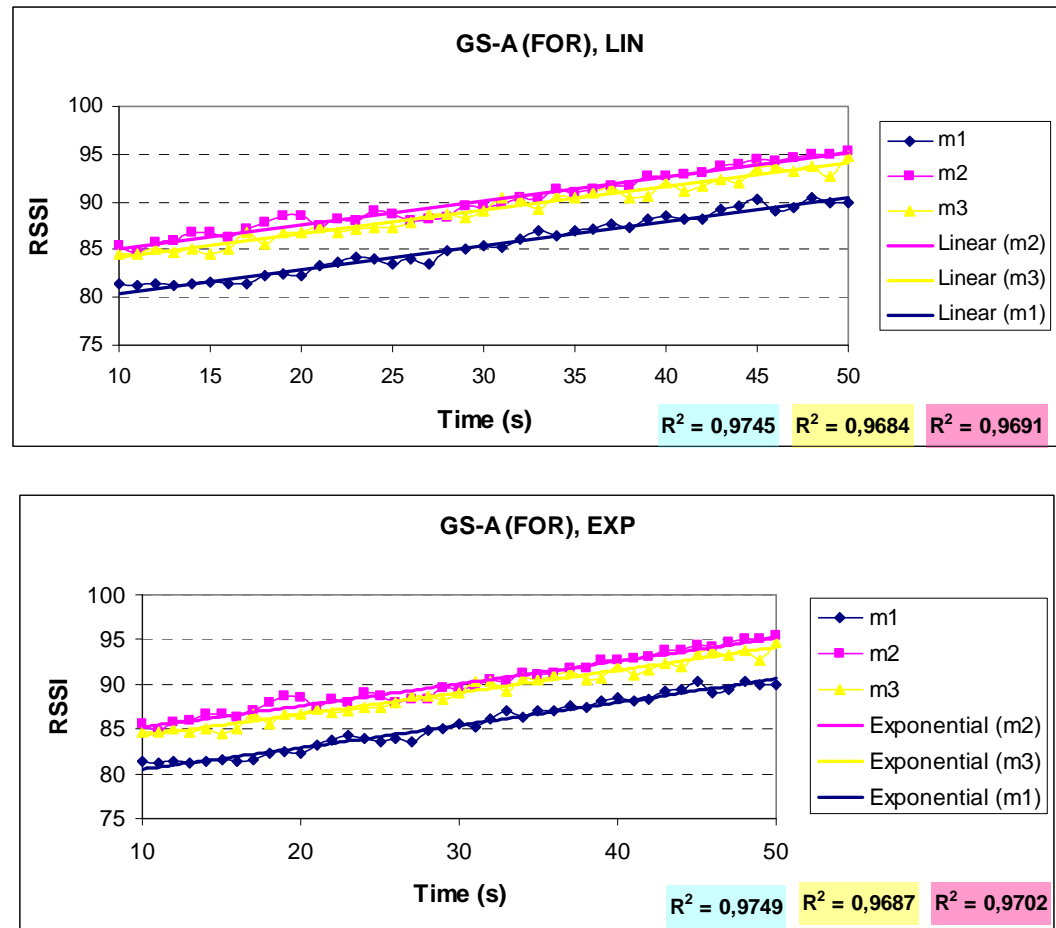

(b)
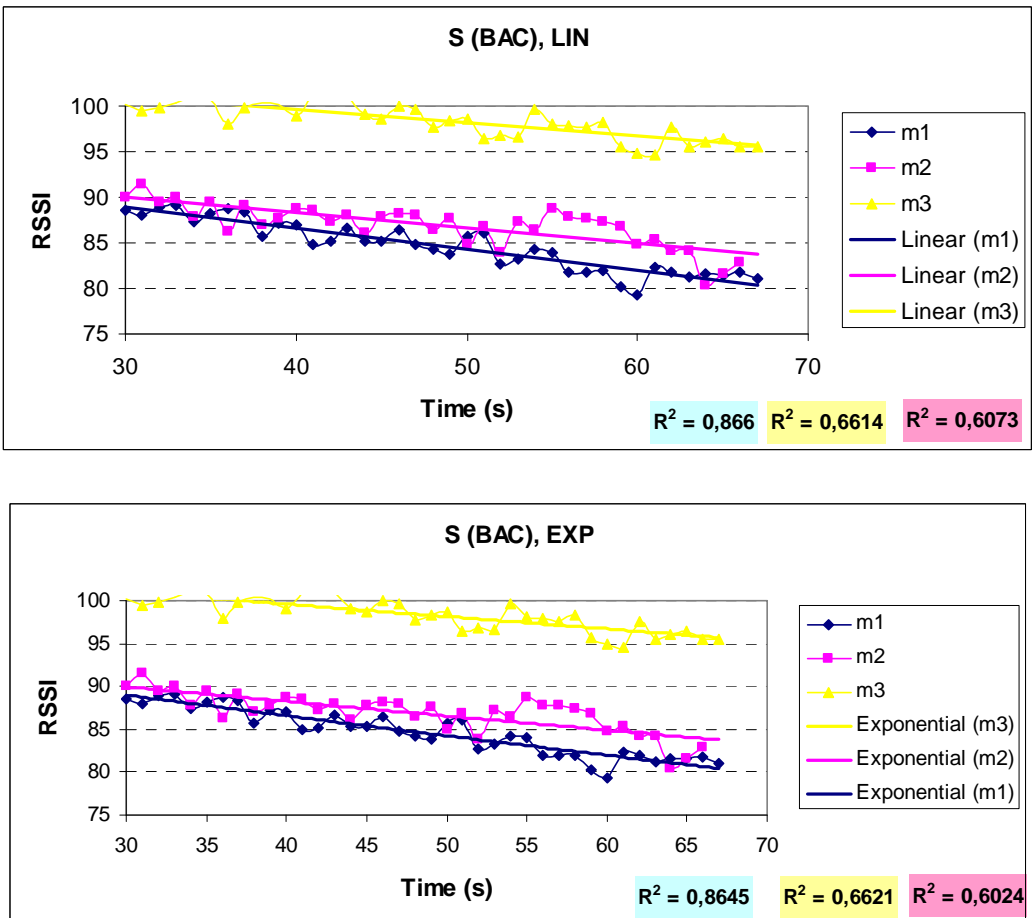

Figure 3: Trends in different paths: a) G-S-A (FOR), b) S (BAC). 
(a)

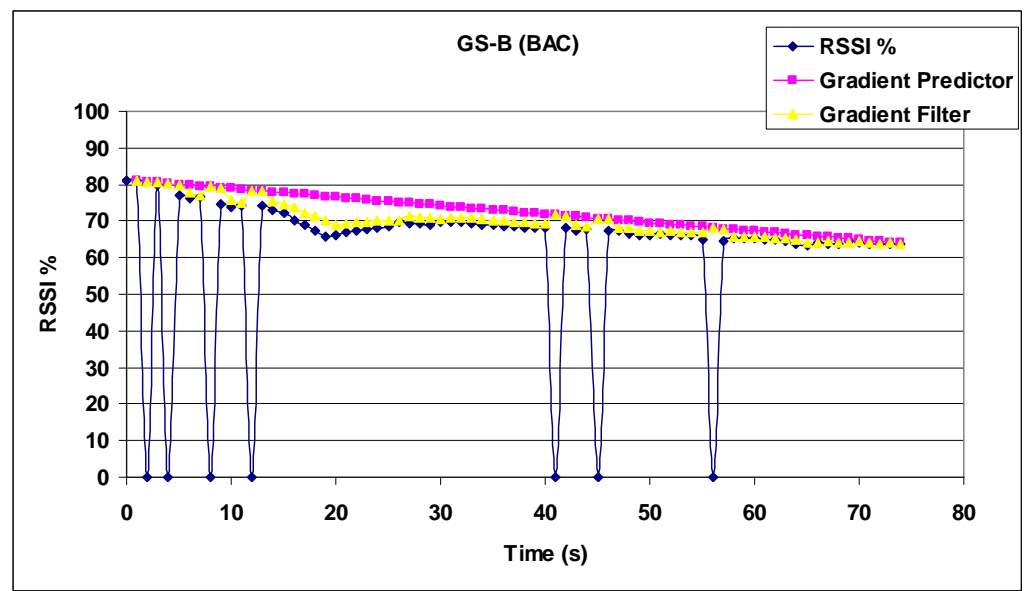

(b)

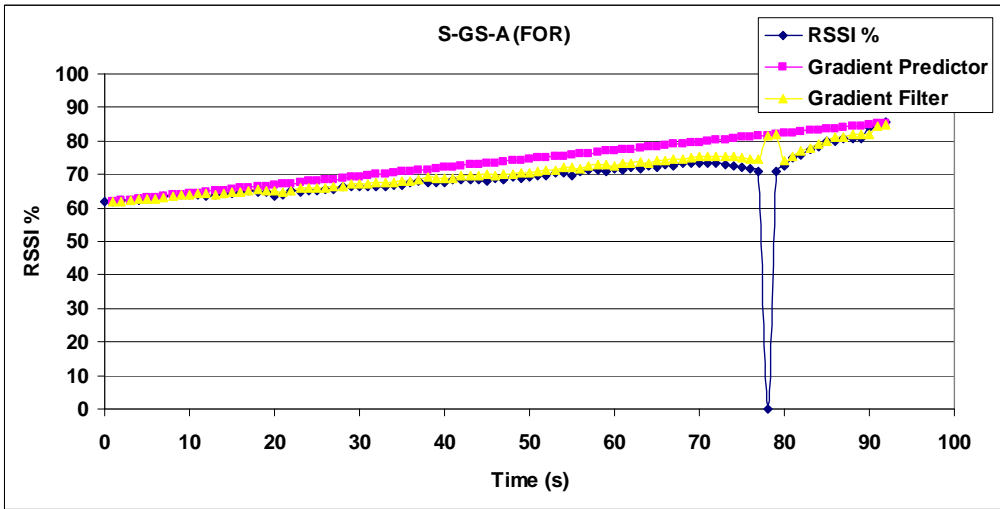

Figure 4: Behavior of the Gradient RSSI filter and predictor: a) with more than one hole at initial instants of time. b) An unexpected value of the filter in instant 78.

\section{Applications of the Gradient RSSI Filter and Predictor to network algorithms and protocols}

The definition of a good filter and predictor for RSSI values is crucial. The importance of a high quality predictor and filter is that RSSI is used for a lot of applications and services of current wireless networks.

In localization applications in all kind of wireless networks (sensors, mobile telephones ...) the RSSI can be used to define a localization indoor system. Generally three APs are needed to define an intersection point inside the coverage area. For these kinds of applications the accuracy of RSSI prediction is important. False predicted values will cause 
bad localization of the MC. For non real time tracking systems the influence of the RSSI predictors and filters are relatively low. In tracking systems also the RSSI is used to follow the path of the MC. A good filter and predictor is needed in this case. We do not use Gradient RSSI filter and predictor for tracking systems. But we have tested a lot of kinds of movements and it is clear it can be used with good results. A Kalman filter for RSSI is used in order to traduce the value of current RSSI into a geographical area value which corresponds with the approximate positioning of the mobile terminal inside the coverage area. In [3] authors use the Extended Kalman filter to train an Artificial Neural Network that is used as well to train the Global System for Mobile communications (GSM) signal strength measurement for mobile positioning which in this technique depends mainly on the GSM feature that can measure the signal strength from many nearby APs. In [12] they convert the measured RSSI values to distances depending on the previous position estimation, then use these distances as inputs for the Kalman Filter that gives the estimation of the next position, also in [13] they apply the Extended Kalman Filter on the distance estimated from converting RSSI values. In [4], the authors use an Extended Kalman Filter for the estimation of location and velocity of a tracked node. They used an improved version to correct the state estimation of Kalman filter because it works well only if the tracked node moves at a constant speed and does not change its direction.

Another range of applications in which RSSI predictors and filters can be used are handover processes. In handover prediction applications a MC is moving along a coverage area and in certain space and time it must change it association to another AP. When MC receives weak signal and need to handover, channel conditions deteriorate, data are lost, and the medium saturates with broadcasted failed data, which cause poor multimedia reproduction quality at the MC [14]. As a result, sporadic and not predicted service disruptions could occur. These kinds of disruptions also can occur inside the coverage area in which signal strength can go down randomly. When MC starts to loss its connection to the associated AP and stops receiving data for several seconds [14], it searches for another AP to associate with. Handover process [15]-[19] is required here to maintain channel connection and frame transmission while MC is moving. As a prediction model, GM plays an important role to make accurate prediction in various fields [5]; by using some measured RSSI values as the data of the GM we can predict next RSSI. In [1] authors apply some filtering techniques including GM to RSSI values to predict handover. In [9] they use grey predictor to predict the RSSI values which are the input of another filter (fuzzy decision system) that produce the handover factor. Different works have been done lately in the area of RSSI filtering. In [5] the authors propose an evaluation of different techniques to filter RSSI values including Discrete Kalman in order to better advance information of the different AP at which handover can be done in an instant of time. They find that Kalman filter mitigates RSSI fluctuation accurately following the current RSSI sequence.

Physical properties of wireless channels make difficult to guarantee the necessary Quality of Service (QoS) [20] for multimedia services. This is true for moving MC or static MC. That is due to they communicate a continuous flow of packets that exhibit a particular semantic and synchronization. Examples of this kind of application are: Video on Demand 
(VoD), Voice over IP (VoIP), and Real Time Chatting. Multimedia service parameters such as: bandwidth, security, traffic shape, packet loss and service connection recovering are influenced by handover. During handover process time, a lot of packets may be lost and the user of MC will not follow the video or audio adequately as in [21]. These parameters are also influenced by holes when the MC is static (really they are important in any case). The high variability of RSSI due to wireless channel characteristics provokes bandwidth variability and cause important loss of performance in multimedia applications. This clearly influences the handover for multimedia applications and also influences the performance of multimedia applications on wireless networks.

We in [22] presented the basic ideas of a new protocol for controlling handover and also the possibility the MC moves to a zone with no coverage previous to complete a handover process and vice versa. We also consider the $\mathrm{MC}$ is receiving a video flow using streaming technique. Next we present the basic steps of the application of the filter and predictor of RSSI (that implement a simple algorithm) for improving this protocol. We did some tests to this basic protocol using GM, Kalman and Gradient RSSI filter and predictor. The best filter and predictor for this application is the Gradient RSSI filter and predictor. It produces the most accuracy and also the best behaviour in presence of holes. The basic entities of our protocol are in table 2 . In the second column the basic actions of the entities are also shown.

Table 2: Basic entities of the handover protocol for streaming multimedia information.

\begin{tabular}{|c|c|}
\hline Entity & Main actions \\
\hline $\begin{array}{c}\text { Worldwide Interoperability for } \\
\text { Microwave Access (WiMAX) Base } \\
\text { Station (WBS) }\end{array}$ & $\begin{array}{l}\text { It is connected to Internet and to a } 2 \text { WiFi APs. In } \\
\text { Internet there is a video server that is in charge to send } \\
\text { multimedia information to the WBS using Real Time } \\
\text { Protocol and Real Time Streaming Protocol. }\end{array}$ \\
\hline WiFi AP & $\begin{array}{l}\text { There are } 2 \text { WiFi APs connected to the WBS. They } \\
\text { receive video from the WBS and send it to the MC. }\end{array}$ \\
\hline$M C$ & $\begin{array}{c}\text { It is moving along the coverage area of the } 2 \mathrm{WiFi} \text { APs. } \\
\text { This means sometimes they must start a handover } \\
\text { process. Using the Gradient RSSI filter and predictor } \\
\text { they can assure no loss of multimedia information in } \\
\text { that process. }\end{array}$ \\
\hline AP Proxy & $\begin{array}{l}\text { AP Proxy runs on the APs. It is in charge to forward } \\
\text { signaling information from MC to WBS and data from } \\
\text { WBS to MC. }\end{array}$ \\
\hline WBS Buffer Manager (WBSBM) & $\begin{array}{l}\text { It is in charge to manage a buffer for multimedia } \\
\text { information in the WBS. }\end{array}$ \\
\hline MC Buffer Manager (MCBM) & $\begin{array}{l}\text { It is in charge to manage a buffer for multimedia } \\
\text { information in the MC. }\end{array}$ \\
\hline
\end{tabular}


The actions of the different entities of our protocol are guided by the Gradient RSSI filter and predictor. The basic idea is that the MC is moving along the coverage zones of APs. But also could fall in a hole. When the MC is near to a zone in the limit of the coverage area of any AP the Gradient Filter and predictor raise a special condition in the MC. In this way the MCBM entity can send a message to the BSBM indicating to increase the speed of communication in order to store a greater amount of video in the MC buffer. This is for supporting the future probable disconnection. Moreover, the amount of video is given on the value raised by the Gradient RSSI filter and predictor. Another interesting and novel idea is the fact that the Gradient RSSI filter and predictor do not raise a special condition for holes inside the coverage areas of the APs because. In this way the handover process is not started inappropriately. A final interesting fact is that the MC can discover (using the Gradient RSSI filter and predictor) when it is close to the common area between the APs in which a handover will occur. The good news here is that the MC can differentiate the case when it is entering of leaving that zone and the direction in which it is moving using our filter and predictor.

In order to illustrate in a simple way the main actions of our protocol we present the basic actions of their primitives in table 3.

Table 3: Basic actions of the protocol's primitives.

\begin{tabular}{|c|c|}
\hline Entity & Main actions \\
\hline $\begin{array}{c}\text { Increase/Decrease Speed of Video } \\
\text { Frames Transmission }\end{array}$ & $\begin{array}{c}\text { The MCBM (based on Gradient Filter and Predictor } \\
\text { raised special condition) request the WBSBM to } \\
\text { increase/decrease the speed of video transmission } \\
\text { (WBSBM request the same to the video server). }\end{array}$ \\
\hline $\begin{array}{c}\text { Start/Stop Buffering Additional } \\
\text { Frames }\end{array}$ & $\begin{array}{l}\text { The MCBM/WBSBM start/stop a process in which they } \\
\text { proactively store video frames. This action is based in } \\
\text { the Gradient filter and predictor special condition } \\
\text { raised. Let us note that disconnection is predicted by our } \\
\text { predictor proactively. }\end{array}$ \\
\hline Predict Disconnection Time & $\begin{array}{l}\text { The power of our filter and predictor is that it allows } \\
\text { predicting the exact time in which the MC will be out of } \\
\text { coverage supposing it moves at constant speed. The MC } \\
\text { can start this primitive in order to discover an amount of } \\
\text { time in which it will be out of coverage. It is important to } \\
\text { remember that our filter and predictor differentiate } \\
\text { clearly a hole or handover state from a disconnection. }\end{array}$ \\
\hline Manage Proactive Handover & $\begin{array}{l}\text { Our filter allows the MC to communicate to the APs } \\
\text { (and those to the WBS) the situation in which a } \\
\text { handover will be start after some time. But also it can } \\
\text { inform when a handover must be aborted before to } \\
\text { finish it. This allows a better storing of multimedia } \\
\text { information (buffer optimization). }\end{array}$ \\
\hline
\end{tabular}



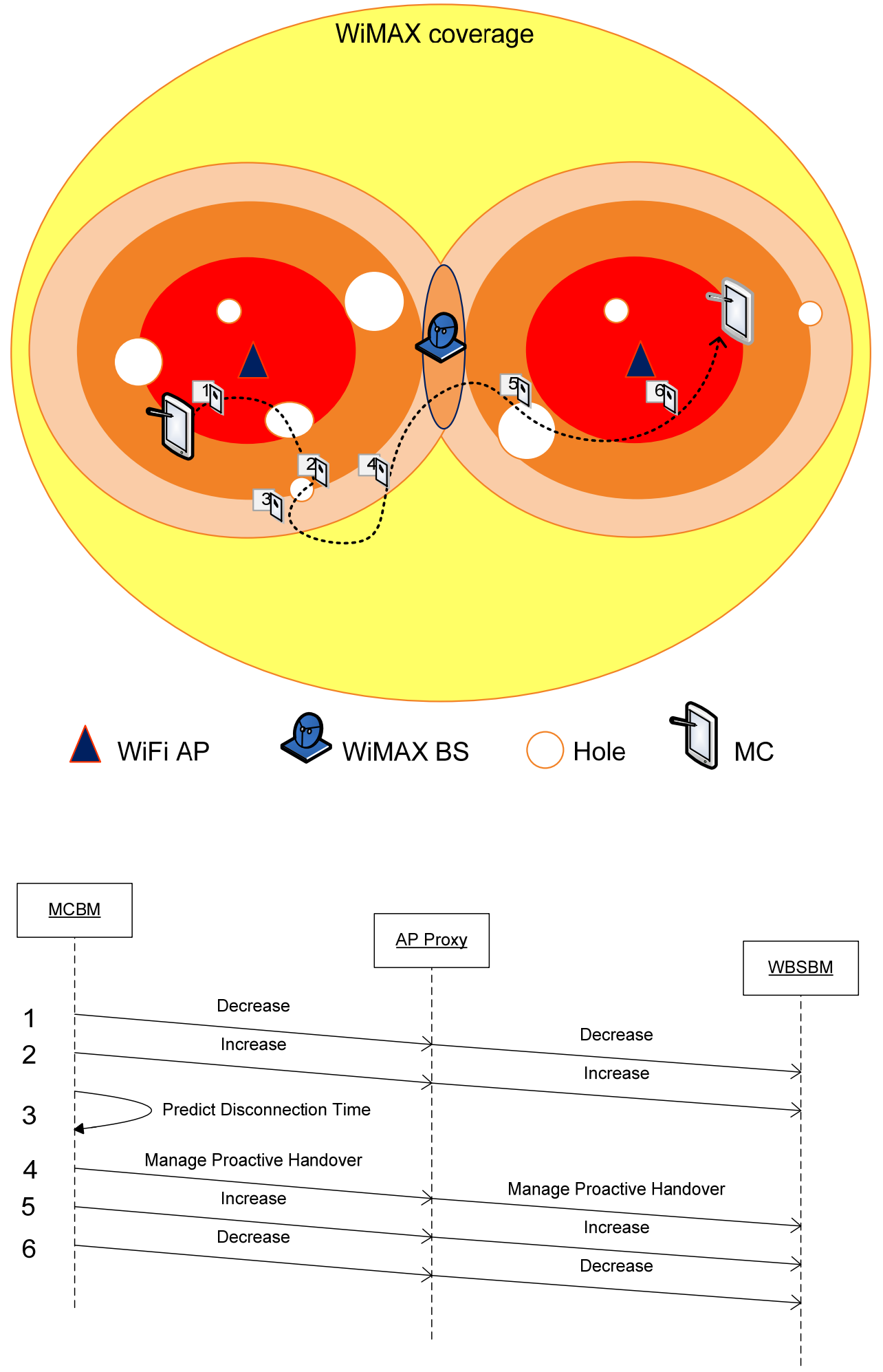

Figure 5: An example of MC movement and the protocol primitives it issues. 
Next we present an example of MC trajectory in order to illustrate the coordination of the above primitives. Let us note that we only will specify the sending's primitives but not their response. That is, we only are interested in to show how the MC can (in coordination with the APs and WBS) controls different primitives along it movement. We will not indicate the actions of the video server. In Figure 5 we insert some numbers in the trajectory of the MC to facilitate the actions of the MC (approximately). As it is shown, the coverage area consist in a big WiMAX coverage area in which there are two coverage areas of the WiFi APs and a handover zone between both APs. Inside the coverage areas of the WiFi APs are different levels of signals and also a number of holes. As it is shown in point number 1 the MC is moving from a zone with low of RSSI to a zone with a higher level of RSSI. In that moment the Mc obtain a response of the Gradient filter of RSSI indicating that situation and then it indicate the WiFi AP to decrease the speed of video transmission as indicated in the signaling picture in the bottom part of the Figure 5. In point number two the MC issues an Increase primitive due to it is suffering decrease values of RSSI's values in the gradient Filter (let us note that the hole in point number 2 is well filtered because the MC is not proposing the stopping the video transmission to the $\mathrm{AP}$ ). In point number 3 the $\mathrm{MC}$ start the Gradient Predictor to discover when it will be out of coverage and to follow increasing the spped of video transmission. In Point number 4 the MC discovers it is closed to a handover zone and then it issues a proactive handover primitive. This primitive was issued successfully and this allows the Mc to cross the handover zone without problems (when it arrives to the coverage area of the other WiFi AP it can continue receiving video normally). Points 5 and 6 are similar to the points 2 and 1 respectively. Let us note that hole in point number 5 is filtered adequately.

\section{Conclusion}

The expansion of wireless networks in the last years is spectacular. They have been implemented in several scenarios: home, office, villages ... We centered our attention to outdoor wireless communications. The well known problems of wireless communications make the MC could experiment some problems of communication. The wireless channel varies along time and space making the bandwidth to be also very variable. But the principal problem is that MC could suffer the problem of disconnection from the AP. That is, the MC could interrupt it communication with the others MC. This is a very important problem in several scenarios, for example in localization system this means the MC could not be localized during the disconnection, in handover process this means the $\mathrm{MC}$ will not connect again in the deadline established and finally in a multimedia information delivery system this means the MC will suffer service disruptions. These disruptions affect the QoS of communications.

The control of disconnections could be achieved modeling the filtering and prediction of RSSI in an appropriate way. In this paper we have tested the synthetic performance of three filters. Our filter and predictor exhibits the best performance because it contemplates holes of coverage inside the coverage area. The control of holes is very important due to they lead to 
sporadic multimedia service disruptions.

The experimental results we present in this paper indicate our filter and predictor behaves very well in practice and that holes appear in practice frequently. We plan to improve the behavior for particular movement cases.

\section{Acknowledgement}

This work has been partially supported by the Departament of Telematic Engineering, and the University of Las Palmas de Gran Canaria.

\section{References}

[1] Bellavista P., Corradi A. And Giannelli C., Evaluating filtering strategies for decentralized handover, Proc. $11^{\text {th }}$ IEEE Symposium on Computers and Communications, Pula-Cagliari, Sardinia, Italy, 2006, 167-174.

[2] Leoni M., Humayoun S. R., Mecella M. and Russo R., A Bayesian approach for disconnection management in mobile ad-hoc networks, Ubiquitous Computing and Communication (UBICC) Journal - Special Issue - CPE, 2008.

[3] Anne K.R., Kyamakya K., Erbas F., Takenga C. and Chedjou J.C., GSM RSSI-based positioning using extended Kalman filter for training artificial neural networks, Proc. of the IEEE Vehicular Technology Conference, Los Angeles, California, USA, 2004, 4141 -4145 .

[4] Kusy B., Ledeczi A. And Koutsoukos X., Tracking mobile nodes using RF Doppler shifts, Proc. of the $5^{\text {th }}$ Iinternational Conference on Embedded Networked Sensor Systems, Sydney, Australia, 2007, 29 - 42.

[5] Li G.D., Yamaguchi D., Mizutani K. and Nagai M., New proposal and accuracy evaluation of grey prediction GM, Oxford journals, IEICE Transactions on Fundamentals of Electronics, Communications and Computer Sciences, E90-A(6), 2007, 1188-1197.

[6] Trestian R., Ormon O. and Muntean G.-M., Signal Strength-based Adaptive Multimedia Delivery Mechanism, IEEE 34th Conference on Local Computer Networks, pp: 297-300, 2009.

[7] Atalah K., Macias E. and Suarez A., RSSI Gradient: New Predictor And Filter To Support Sporadic Wireless Service Interruptions, Seventh IASTED International Conference on Communication Systems and Networks, pp: 70-78, 2008.

[8] Bellavista P., More details about Grey Model, http://lia.deis.unibo.it/research/SOMA/SmartBuffer/Client/htmIDocs/detailsMobPred.ht ml, available June 2010. 


\section{Macrothink}

Network Protocols and Algorithms

ISSN 1943-3581

2010, Vol. 2, No. 2

[9] Lee C.H. and Yu C.J., An Intelligent handoff algorithm for wireless communication systems using grey prediction and fuzzy decision system, IEEE International Conference on Networking, Sensing and Control, Taipei, Taiwan, 2004, 541- 546.

[10] Welch G. and Bishop G., An Introduction to the Kalman Filter, ACM SIGGRAPH, 2001.

[11] Toh C.K., The design \& implementation of a hybrid handover protocol for multimedia wireless LANs, Proc. of the First Annual ACM International Conference on Mobile Computing and Networking, Berkeley, California, USA, 1995, 49-61.

[12] Helén M., Latvala J., Ikonen H. and Niittylahti J., Using calibration in RSSI-based location tracking system, TechRepublic White Paper, 2007.

[13] Kotanen A., Hännikäinen M., Leppäkoski H. and Hämäläinen T. D., Positioning with IEEE 802.11b wireless LAN, $14^{\text {th }}$ IEEE International Symposium on Personal, Indoor and Mobile Radio Communications, Beijing, China, 2003, 2218-2222.

[14] Vilas M., Pañeda X. G., Melendi D., García R. and García V. G., Signaling management to reduce roaming effects over streaming services, IEEE Proceedings of the $32^{\text {nd }}$ EUROMICRO Conference on Software Engineering and Advanced Applications, Cavtat, Dubrovnik, Croatia, 2006.

[15] Cho H.D., Park J.K., Ko W.J., Lim K. and Kim W., A study on the MCHO method in hard handover and soft handover between WLAN and CDMA, International Conference on Consumer Electronics ICCE 2005, Digest of Technical Papers, Las Vegas, USA, 2005, 391- 392.

[16] Choi H.H. and Cho D.H., Takeover: a new vertical handover concept for next-generation heterogeneous networks, IEEE $61^{\text {st }}$ Vehicular Technology Conference, 4, Stockholm, Sweden, 2005, 2225-2229.

[17] Corvaja R., Zanella A., Dossi M., Tontoli A. and Zennaro P., Experimental performance of the handover procedure in a WiFi network, Proc. of WPMC04, Abano Terme, Italy, 2004.

[18] Li M., Sandrasegaran K. and Tung T., A Multi-interface proposal for IEEE 802.21 Media Independent Handover, International Conference on the Management of Mobile Business (ICMB 2007), Toronto, Canada, 2007, 7-7.

[19] Dong G. and Dai J., An improved handover algorithm for scheduling services in IEEE8O2.16e, IEEE Mobile WiMax Symposium, Orlando, Florida, USA, 2007, 38-42.

[20] Guo Ch., Guo Z., Zhang Q. and Zhu W., A seamless and proactive end-to-end mobility solution for roaming across heterogeneous wireless networks, IEEE Journal on Selected Areas in Communications, 22, 2004, 834-848.

[21] CDMA Development Group, CDMA2000 international roaming: a status report, 2005. 


\section{Macrothink}

Network Protocols and Algorithms ISSN 1943-3581 2010, Vol. 2, No. 2

[22] Suárez A., Elbatsh K. and Macias E., A Proactive horizontal handover algorithm for WiFi-WiMax Interoperable Networks, The 2007 International Conference of Wireless Networks, London, U.K., 2007, 1494-1499 